\title{
CARACTERIZACIÓN DE LOS CONCEJOS ASTURIANOS A PARTIR DE SU PARTICIPACIÓN EN LA ECONOMÍA SOCIAL
}

\author{
Aydee Hurtado Garcés \\ Departamento de Economía Aplicada. Universidad de Oviedo \\ hurtadoaydee.uo@uniovi.es \\ Carmen Ramos Carvajal \\ Departamento de Economía Aplicada. Universidad de Oviedo \\ cramos@uniovi.es
}

\section{RESUMEN}

La Economía Social (ES) es un sector relevante para la economía, pero a pesar de ello, la información disponible sobre dicho sector es escasa. El objetivo fundamental de este trabajo consiste en caracterizar espacialmente los concejos asturianos a partir de su participación en la Economía Social. Para ello, se han determinado diversos indicadores, entre los que se encuentran los coeficientes de localización y especialización de las actividades en los distintos concejos considerando la relación entre la actividad de la entidad y su localización. Además, se obtienen unos índices comparativos de la ES, cooperativas y sociedades laborales a través del Análisis de Componentes Principales.

Palabras clave: Análisis de Componentes Principales, Cociente de Localización, Coeficiente de Especialización, Análisis de la Varianza, Economía Social, Cooperativas y Sociedades Laborales.

\section{ABSTRACT}

The Social Economy (SE) is an important sector for the economy; however, there is little information on this sector. This work analyzes, spatially, entities of the Asturian SE on two levels: one-dimensional and multidimensional. In the first, shall be made a study of localiza-

Fecha de recepción: febrero 2012.

Fecha de aceptación: diciembre 2012. 
tion and specialization of activities in the various councils and will analyze the relationship between economic activity and its location. In the multivariate analysis will obtain indexes to compare the SE, cooperatives and labor societies in different Asturian councils through the Principals Components Analysis.

Key words: Principal Component Analysis, Location Quotient, Coefficient of Specialization, Analysis of Variance, Social Economy, Cooperatives and Labor Societies.

\section{INTRODUCCIÓN}

El término «Economía Social» ha sido un concepto ampliamente discutido, a lo largo de la historia han sido diversos los términos que han surgido para hacer referencia a aquellas entidades que no están encuadradas ni en el sector público ni en el sector privado capitalista, como tercer sector, tercer sistema, economía solidaria, entre otros. No obstante, pese a que todas estas denominaciones intentan describir realidades similares, la delimitación del campo de actividad no siempre ha sido la misma, pudiendo diferir de un enfoque a otro e incluso, dentro de un mismo enfoque, esta delimitación puede variar en el tiempo o en la región donde se utilice.

Durante los últimos años la Economía Social ha adquirido una gran importancia en el desarrollo económico de las regiones, debido en gran medida a las características propias de las entidades que la componen, que influyen de manera significativa en magnitudes como el rendimiento económico, la capacidad de generación de empleo y el aumento en el bienestar social. Evolución que ha estado unida al creciente interés de investigadores, gobiernos y organismos supranacionales, como la Unión Europea, en analizar su importancia en las regiones e implementar políticas encaminadas a reforzar este sector.

Dada la importancia del Sector de la Economía Social (SES en adelante) a nivel económico, social y medioambiental y la amplia discusión académica sobre su delimitación, se aprueba recientemente en España la Ley de Economía Social ${ }^{1}$, según la cual, este sector está compuesto por cooperativas, sociedades laborales, mutualidades, empresas de inserción, centros especiales de empleo, cofradías de pescadores, sociedades agrarias de transformación, fundaciones, asociaciones y entidades singulares creadas por normas específicas que se rijan por los principios establecidos en esta Ley. Ahora bien, de acuerdo a la actividad y función principal de las entidades se pueden dividir en dos subsectores, sector empresarial o de mercado y sector no empresarial o de no mercado; al primer grupo le corresponden todas las entidades enumeradas anteriormente a excepción de las tres últimas, pertenecientes al sector no empresarial ${ }^{2}$.

Pese a su importancia, hay poca información estadística sobre el SES, siendo hasta la fecha difícil disponer de informes periódicos que permitan conocer en detalle sus principales agregados macroeconómicos y su grado de desarrollo. Esta dificultad se acrecienta si el objetivo es realizar análisis regionales, pues tal como están construidas las Cuentas Nacionales y

1 Véase Ley 5/2011, de 29 de marzo, de Economía Social.

2 En Monzón et al. (2010) se presenta una clasificación detallada del SES. 
Regionales, las correspondientes a este sector se encuentran dispersas en los distintos sectores de la economía, y de ahí la dificultad para su medición.

Como en otras regiones españolas, en Asturias, el SES tiene una importante representación, según datos publicados por la Dirección General de Fomento de la Economía Social y del Fondo Social Europeo y los distintos registros relacionados con el sector, en el ejercicio 2005 existían un total de 5.088 entidades, de las cuales 952 (18,7\%) corresponden al subsector de mercado. En contra de la menor representación de este último subsector, tienen un fuerte impacto en términos económicos y en la generación de empleo. Así, del total de 12.052 empleos directos del SES, más de la mitad (en concreto el 67,4\%) corresponden a este sector de mercado. Este fuerte dinamismo resulta principalmente de la presencia de las sociedades cooperativas y sociedades laborales en la región.

El objetivo de este artículo consiste en caracterizar los diferentes concejos de la comunidad asturiana desde el punto de vista de su participación en la Economía Social. Dado que se considera que la perspectiva espacial es fundamental en los estudios económicos, se pretende efectuar un estudio que considere tal óptica. Con el objeto de llevar a cabo este estudio, se efectúa un doble análisis aplicando los enfoques unidimensional y multidimensional. Desde el primero de ellos, se determinan coeficientes que permitan describir los concejos asturianos a partir de su especialización productiva y de la localización en los mismos de empresas de distintas ramas de actividad económica. Desde la óptica multidimensional, se caracteriza a los municipios mediante el análisis de su estructura económica en relación a su participación en la Economía Social.

Este trabajo se distribuye como sigue: en la segunda sección se describe la información estadística empleada. En el tercer apartado se realiza en primer lugar, un análisis espacial a partir de diversos indicadores de localización y especialización y, en segundo lugar, un análisis de la varianza, con el fin de determinar sí la localización influye en el tipo de actividad económica de las entidades analizadas. En la cuarta sección, se caracterizan a los concejos mediante la aplicación del análisis de componentes principales. Finalmente, en la última parte se recogen las conclusiones más relevantes alcanzadas en esta investigación.

\section{INFORMACIÓN ESTADÍSTICA}

La información estadística necesaria para efectuar este estudio se extrae de las cuentas satélite ${ }^{3}$ de la Economía Social Asturiana, (Hurtado et al. 2012) a partir de la información obtenida en el registro de cooperativas, registro mercantil y publicaciones oficiales del Ministerio de Trabajo e Inmigración. Para el análisis del conjunto de la Economía Social, se han tenido en cuenta todas las entidades incluidas en la citada Ley pertenecientes a la economía de mercado, a saber: cooperativas (213 empresas), sociedades laborales (497 empresas), mutualidades (2), sociedades agrarias de transformación - SAT (33), cofradías de pescadores (2), centros especiales de empleo - CEE (52) y las empresas de inserción (2). Adicionalmente, se realiza un análisis comparativo con los dos grupos más relevantes del sector de mercado de la Economía Social, las cooperativas y las sociedades laborales, dicha importan-

3 Las cuentas satélite son un conjunto de tablas que ofrecen información más detallada y flexible de una determinada realidad económica o social que la presentada en su marco de referencia por la Contabilidad Nacional. 
cia se manifiesta en términos económicos (con una participación en la producción del 78,3\% en el total del sector de mercado) y en el nivel de empleo (que alcanza una peso del $67,4 \%$ ).

Con el objetivo de determinar los coeficientes de localización y especialización se considera el nivel de empleo del SES y del total de la economía asturiana por sector de actividad. Por otra parte, para efectuar la caracterización de los concejos, se consideran 43 variables macroeconómicas, de las cuales se han utilizado 12, por su mejor comportamiento. La descripción de estas últimas se puede encontrar en la Tabla $1^{4}$.

Tabla 1

VARIABLES UTILIZADAS EN EL ANÁLISIS

\begin{tabular}{|c|c|c|}
\hline Variable & Nombre & Observaciones \\
\hline CI & Consumos intermedios & \multirow{9}{*}{$\begin{array}{l}\text { Variables cuantitativas expresadas en } \\
\text { euros. }\end{array}$} \\
\hline $\mathbf{R A}$ & Remuneración de asalariados & \\
\hline RPpag & Rentas de la propiedad pagadas & \\
\hline PS & Prestaciones sociales & \\
\hline FBKF & FBKF & \\
\hline CK & Consumo de Capital & \\
\hline Pn & Producción & \\
\hline RPrec & Rentas de la propiedad recibidas & \\
\hline OTCrec & Otras transferencias recibidas & \\
\hline Sector 2 & Sector secundario & \multirow{2}{*}{$\begin{array}{l}\text { Proporción de entidades pertenecientes a } \\
\text { un determinado sector de actividad. }\end{array}$} \\
\hline Sector3 & Sector terciario & \\
\hline Empleo & Empleo & Número de empleados. \\
\hline
\end{tabular}

Fuente: Elaboración propia.

Es preciso señalar, que para llevar a cabo el estudio basado en los cocientes de localización y especialización se analizan 53 concejos de los 78 existentes en el Principado de Asturias; no obstante, para el análisis multivariante de componentes principales se tienen en cuenta únicamente los 34 municipios en los que las cooperativas y sociedades laborales están representadas.

\section{CARACTERIZACIÓN DE LOS CONCEJOS ASTURIANOS}

Como punto de arranque de la caracterización de los de los concejos ${ }^{5}$ asturianos, se analiza si la ubicación geográfica influye en la realización de una actividad económica u otra,

4 Para efectuar la caracterización mediante análisis multivariante, se ha excluido el sector de actividad primario, ya que no refleja correlación con el resto de variables del modelo y presenta, además, una representatividad muy pequeña, por lo que no se justifica su inclusión.

5 Es preciso señalar que el término concejo en el Principado de Asturias equivale a municipio. 
por parte de las empresas de Economía Social. Para ello, se va a efectuar un análisis de la varianza, con un único factor, considerando la realización de una actividad económica como variable dependiente (variable efecto) y la situación geográfica como factor (variable causa), del cual las modalidades serían las 8 comarcas asturianas ${ }^{6}$.

Para llevar a cabo un análisis de la varianza es preciso comprobar, previamente, si se verifican una serie de supuestos, entre los que se encuentran la normalidad de la variable y la homogeneidad de las varianzas para cada nivel del factor. Ambas condiciones se cumplen ${ }^{7}$, por lo tanto, la realización de un análisis de la varianza es correcta y se procede a su realización, cuyos resultados se muestran en la Tabla 2.

Tabla 2

INFLUENCIA DE LA SITUACIÓN GEOGRÁFICA EN LA REALIZACIÓN DE LAACTIVIDAD ECONÓMICA

\begin{tabular}{|l|r|r|r|c|c|}
\hline & Suma de cuadrados & \multicolumn{1}{c|}{ gl } & Media cuadrática & F & \multicolumn{1}{c|}{ Sig. } \\
\hline Inter-grupos & 1657521,796 & 7 & 236788,828 & 2,120 &, 080 \\
\hline Intra-grupos & 2680102,411 & 24 & 111670,934 & & \\
\hline Total & 4337624,206 & 31 & & & \\
\hline
\end{tabular}

Fuente: Elaboración propia.

A la vista de los resultados obtenidos en la tabla anterior, donde se muestra el rechazo de la hipótesis nula del contraste, ya que el nivel de significación está por debajo de 0,1 , por lo tanto se puede concluir que la ubicación geográfica influye en la actividad económica realizada por las empresas de Economía Social.

Se ha repetido este análisis para las cooperativas y para las sociedades laborales, no hallándose ninguna evidencia en este sentido. Esto es, se ha derivado que la situación geográfica no influye en las actividades llevadas a cabo por las cooperativas y sociedades laborales, aunque sí existe en la Economía Social en su conjunto. Consideramos que esto se debe a que dentro del concepto de Economía Social se incluyen, entre otras entidades, las cofradías de pescadores y las sociedades agrarias de transformación, las cuales dado su especial carácter ligado a la localización geográfica «sesgan» los resultados en ese sentido, provocando la existencia de tal relación.

\subsection{Coeficiente de especialización}

El estudio de la localización sectorial y el análisis de su nivel de especialización en las distintas regiones, aporta información de especial relevancia para la toma de decisiones en los ámbitos político, social y económico. Durante los últimos años han sido muchos los tra-

6 El Principado de Asturias está dividido en ocho comarcas funcionales o áreas de planificación territorial, a saber: 1. Comarca de Avilés (10 concejos), 2. Comarca del Caudal (3 concejos), 3. Comarca del Eo-Navia (17 concejos), 4. Comarca de Gijón (3 concejos), 5. Comarca del Nalón (5 concejos), 6. Comarca del Narcea (5 concejos), 7. Comarca de Oriente (14 concejos), 8. Comarca de Oviedo (21 concejos).

7 Se han llevado a cabo los contrastes de Kolmogorov-Smirnov de normalidad y de Levene de igualdad de varianzas, en ambos casos el contraste la hipótesis no sido rechazada. 
bajos empíricos desarrollados sobre este tema, entre los que destacan Beyers (1991), Beyers y Lindahl (1996), Coffey y Shearmour (1997), Henderson (1997 y 2003), Ingram (1998) y más recientemente, Alonso et al. (2004), Desmet y Fafchamps (2005), Gaigné et al. (2005), Polèse y Shearmur (2004 y 2006), Rubiera (2005) y Terral y Polèse (2006).

El análisis de localización y especialización sectorial puede realizarse a partir de distintas variables, como el número de entidades, el volumen de producción o el nivel de empleo. En concreto, aquí se ha tomado esta última variable, dada su importancia en el desarrollo económico y social de las regiones. Además, hay una extensa literatura empírica que analiza la localización de las actividades económicas a partir del nivel de empleo, como en Lemelin y Polèse (1993), Dávila (2004) y Angoa et al. (2009).

El coeficiente de especialización permite determinar qué concejos están especializados en alguna actividad económica concreta y cuáles no lo están.

La fórmula de cálculo es ahora la siguiente:

$$
C E_{r}=\frac{1}{2} \sum_{s}\left|\frac{x_{s r}}{x_{r}}-\frac{x_{s}}{x}\right|
$$

Donde $\mathrm{x}_{\mathrm{sr}}$ representa el empleo de la actividad s en el concejo $\mathrm{r} ; \mathrm{x}_{\mathrm{s}}$ es el empleo del sector de actividad $\mathrm{s}$ en el total regional; $\mathrm{x}_{\mathrm{r}}$ representa el empleo total en el concejo $\mathrm{r} \mathrm{y} \mathrm{x}$ el empleo total a nivel regional.

Esta expresión se encuentra acotada entre 0 y 1 . A medida que se aproxime a la unidad más especializado se encuentra un determinado concejo, y viceversa si se aproxima a 0.

En la Figura 1 se pueden observar los coeficientes de especialización del SES en la región ${ }^{8}$. Los concejos más especializados en el SES son los de Morcín, Aller, Taramundi, Muros de Nalón, El Franco, Coaña y Nava; y entre los menos especializados se encuentran concejos de especial relevancia como Gijón, Oviedo, Avilés y Valdés.

Por tipo de sociedad, el mayor grado de especialización del nivel de empleo generado por las cooperativas se concentra en los concejos de Vegadeo, Ribadesella, Cudillero, Laviana, San Martín del Rey de Aurelio, Taramundi y Nava. Y en las sociedades laborales, los concejos de Allande, Peñamellera Alta, Morcín, Aller, Muros de Nalón y Nava (Tabla 3).

\subsection{Cociente de localización ${ }^{9}$}

El cociente de localización zonal mide el peso relativo que tiene una determinada actividad económica o sector en una región (concejo en nuestro caso). Como ya se ha señalado, la variable analizada es el nivel de empleo de los distintos sectores de la Economía Social en cada uno de los concejos asturianos, respecto al mismo porcentaje a nivel regional.

La expresión matemática que permite cuantificar dicho cociente es la siguiente:

$$
L_{s r}=\frac{x_{s r} / x_{r}}{x_{s} / x}
$$

8 El mapa con la situación y denominación de los concejos se muestra en el Anexo A1.

9 Véase Polèse (1994). 


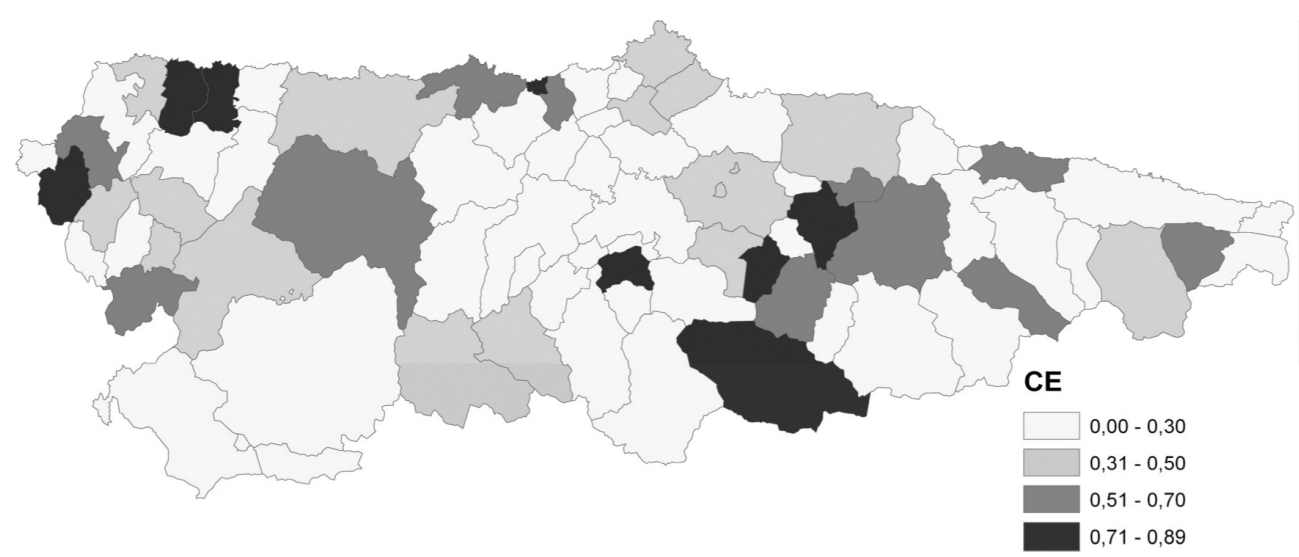

Fuente: Elaboración propia.

Valores de este indicador sobre la unidad, indican que la actividad s se encuentra localizada en el concejo $r$, por encima de la media de la región y, por tanto, en dicho concejo $r$ existirá una localización de empresas que lleven a cabo la actividad s.

En la Figura 2 se puede observar el cociente de localización del SES según rama de actividad y concejo, desde el punto de vista del nivel de empleo. En este sentido, los concejos de Corvera de Asturias, Mieres, Oviedo, Soto del Barco y Coaña presentan la mayor concentración de empleados del sector primario, respecto a la media presentada a nivel regional. El nivel de empleo del sector industrial se localiza principalmente en los concejos de Vegadeo, El Franco, Taramundi, Tineo, Valdés y Tapia de Casariego en el occidente de la región; los concejos de Laviana, Cudillero, San Martín del Rey Aurelio, Muros de Nalón y Oviedo en la zona Centro y Peñameñera Alta, Ribadesella y Parres en la zona oriente. En cuanto al sector de la construcción, destacan los concejos de Morcín, Allande, Aller, Cabrales y Villaviciosa, con un grado de concentración por encima de la media registrada a nivel regional. Por último, el empleo generado por el sector servicios se concentra principalmente los concejos de Amieva, Cabranes, Piloña y Carreño.

En algunos municipios destaca la concentración de empleo del SES por encima de la media a nivel regional en más de un sector de actividad. Así, los concejos de Mieres, Oviedo, Peñamellera Alta, Gozón, Castropol y Parres presentan una concentración de trabajadores en el sector primario e industrial. En los concejos de Llanera, Avilés y Salas se encuentra localizada la mano de obra mayoritariamente de los sectores primarios y servicios. En Valdés y Tapia de Casariego se localizan principalmente los trabajadores del sector industrial y de servicios. Cudillero y Llanes presentan concentración del empleo en el sector industrial y construcción. En Corvera de Asturias se concentran el empleo del sector primario y construcción. Y, por último, Castrillón, con una concentración de mano de obra del sector de la construcción y servicios.

Como resumen de los resultados anteriormente mostrados se puede señalar que las comarcas de Eo-Navia, Avilés y Oviedo muestran una mayor localización en actividades del 
Tabla 3

COEFICIENTE DE ESPECIALIZACIÓN SEGÚN TIPO DE ENTIDAD

\begin{tabular}{|c|c|c|c|c|c|c|c|}
\hline Concejos & SES & Coop. & SL & Concejos & SES & Coop. & SL \\
\hline Allande & 0,47 & 0,31 & 0,93 & Morcín & 0,89 & 0,00 & 0,89 \\
\hline Aller & 0,86 & 0,00 & 0,86 & Muros de Nalón & 0,83 & 0,00 & 0,83 \\
\hline Amieva & 0,68 & 0,68 & 0,00 & Nava & 0,80 & 0,82 & 0,82 \\
\hline Avilés & 0,22 & 0,29 & 0,20 & Navia & 0,14 & 0,46 & 0,24 \\
\hline Cabrales & 0,41 & 0,00 & 0,41 & Noreña & 0,44 & 0,67 & 0,17 \\
\hline Cabranes & 0,61 & 0,00 & 0,61 & Oviedo & 0,21 & 0,11 & 0,17 \\
\hline Cangas del Narcea & 0,29 & 0,41 & 0,37 & Parres & 0,26 & 0,24 & 0,37 \\
\hline Cangas de Onís & 0,20 & 0,33 & 0,00 & Peñamellera Alta & 0,52 & 0,52 & 0,97 \\
\hline Carreño & 0,49 & 0,55 & 0,30 & Pesoz & 0,33 & 0,33 & 0,00 \\
\hline Castrillón & 0,19 & 0,00 & 0,25 & Piloña & 0,55 & 0,54 & 0,57 \\
\hline Castropol & 0,14 & 0,50 & 0,70 & Ponga & 0,00 & 0,00 & 0,00 \\
\hline Coaña & 0,79 & 0,00 & 0,00 & Pravia & 0,24 & 0,60 & 0,20 \\
\hline $\begin{array}{l}\text { Corvera de Astu- } \\
\text { rias }\end{array}$ & 0,33 & 0,62 & 0,33 & Ribadesella & 0,68 & 0,91 & 0,46 \\
\hline Cudillero & 0,62 & 0,90 & 0,76 & Salas & 0,24 & 0,41 & 0,00 \\
\hline Franco, El & 0,82 & 0,00 & 0,48 & $\begin{array}{l}\text { San Martín del } \\
\text { Rey Aurelio }\end{array}$ & 0,71 & 0,85 & 0,61 \\
\hline Gijón & 0,10 & 0,23 & 0,06 & Siero & 0,34 & 0,34 & 0,33 \\
\hline Gozón & 0,42 & 0,56 & 0,55 & Sobrescobio & 0,22 & 0,62 & 0,00 \\
\hline Grado & 0,20 & 0,24 & 0,37 & Somiedo & 0,42 & 0,00 & 0,42 \\
\hline Grandas de Salime & 0,68 & 0,68 & 0,00 & Soto del Barco & 0,54 & 0,00 & 0,52 \\
\hline Illano & 0,45 & 0,45 & 0,00 & Tapia de Casariego & 0,45 & 0,45 & 0,50 \\
\hline Langreo & 0,40 & 0,72 & 0,31 & Taramundi & 0,83 & 0,83 & 0,00 \\
\hline Laviana & 0,56 & 0,87 & 0,51 & Teverga & 0,49 & 0,00 & 0,49 \\
\hline Lena & 0,29 & 0,00 & 0,29 & Tineo & 0,54 & 0,64 & 0,48 \\
\hline Valdés & 0,33 & 0,41 & 0,41 & Vegadeo & 0,52 & 0,96 & 0,43 \\
\hline Llanera & 0,22 & 0,22 & 0,26 & $\begin{array}{l}\text { Villanueva de } \\
\text { Oscos }\end{array}$ & 0,45 & 0,45 & 0,00 \\
\hline Llanes & 0,17 & 0,00 & 0,21 & Villaviciosa & 0,36 & 0,00 & 0,41 \\
\hline Mieres & 0,26 & 0,28 & 0,49 & & & & \\
\hline
\end{tabular}

* A, B: Agricultura, ganadería, caza y selvicultura; pesca; C,D: Industria; F:Construcción y G a O: Servicios. Fuente: Elaboración propia. 
sector primario. Las entidades de la Economía Social cuya actividad es la construcción se encuentran principalmente localizadas en las comarcas de Oviedo, Narcea, Caudal, Gijón y Oriente. En las comarcas de Gijón y Oriente se encuentran fundamentalmente empresas del sector servicios. Por lo que se refiere al sector secundario, se encuentran empresas con alto nivel de empleo localizadas en Oriente, Eo-Navia, Avilés, Oviedo y Nalón.

En la Tabla A.2 del Anexo, se muestra el valor del cociente de localización, tanto del total del SES, como de cooperativas y sociedades laborales. Comenzando con el sector primario, se aprecia que es una actividad relevante entre las cooperativas de los concejos de Corvera de Asturias $(15,85)$, Oviedo $(11,55)$, Mieres $(9,22)$, Pravia $(7,38)$ y Llanera $(7,04)$; sin embargo, en lo que se refiere a las sociedades laborales, sólo dos concejos muestran coeficientes superiores a la unidad, Somiedo $(1,71)$ y Oviedo $(1,22)$. Ambos tipos de sociedades analizadas presentan coeficientes elevados en relación al sector secundario; por el lado de las cooperativas destacan Vegadeo $(23,44)$, Ribadesella $(11,62)$, San Martín del Rey Aurelio $(6,88)$ y Peñamellera Alta $(6,68)$; este último también destaca en las sociedades laborales con un cociente de localización de 30,67; además de Vegadeo $(11,09)$ y Cudillero $(7,31)$. En el sector de la construcción sobresalen los concejos de Cudillero $(10,19)$ y Laviana $(7,54)$ en las cooperativas y Allande $(14,75)$, Morcín $(8,97)$ y Aller $(7,36)$ en las sociedades laborales. Finalmente, el empleo del sector servicios se localiza principalmente en Amieva $(3,13)$, Piloña $(2,24)$ y Salas $(2,05)$ para el caso de las cooperativas y en Castropol $(3,28)$, Cabranes $(2,57)$, Degaña $(2,38)$, Piloña $(2,31)$, Gozón $(2,22)$, Soto del Barco $(2,08)$ y Tapia de Casariego $(2,02)$ en las sociedades laborales.

\subsection{Consideración de variables económicas. Análisis multidimensional}

Dado que se dispone, como ya se ha señalado, de información relativa a varias magnitudes, nos ha parecido adecuado considerarlas conjuntamente para completar la caracterización de los concejos, a través de la realización de un análisis multidimensional. Para llevar a cabo dicho estudio se aplica el Análisis de Componentes Principales (ACP).

Uno de los requisitos necesarios para poder aplicar dicha técnica es la consideración de la matriz de correlaciones, con la finalidad de conocer el grado de correlación existente entre las variables utilizadas en el estudio. Para determinar este aspecto las pruebas más utilizadas son los coeficientes derivados de la matriz de correlación y el determinante asociado a la misma. En este caso, los coeficientes de correlación entre las variables resultaron, en general, bastante elevados, con unos niveles de significación en su mayoría adecuados y un determinante cercano a cero, lo cual indica una elevada correlación lineal entre estas variables y, por tanto, el análisis se puede realizar a partir de un número menor de magnitudes.

Además de las pruebas antes mencionadas, también se han aplicado el test de esfericidad de Bartlett (Bartlett, 1954), donde se contrasta la hipótesis de igualdad entre la matriz de correlaciones y la matriz identidad, y la medida de adecuación de la muestra Kaiser-MeyerOlkin (KMO), que se basa en la comparación de los elementos de la matriz de correlaciones con los coeficientes de correlación parcial. Toma valores entre 0 y 1 , valores cercanos a cero desaconsejan la utilización del análisis de componentes principales ${ }^{10}$.

10 Kaiser (1974) considera un nivel de este índice mayor a 0,9 como muy bueno; entre 0,8 y 0,7 como bueno; entre 0,7 y 0,6 como mediocre; entre 0,6 y 0,5 como bajos y los valores inferiores a 0,5 como muy bajo. 


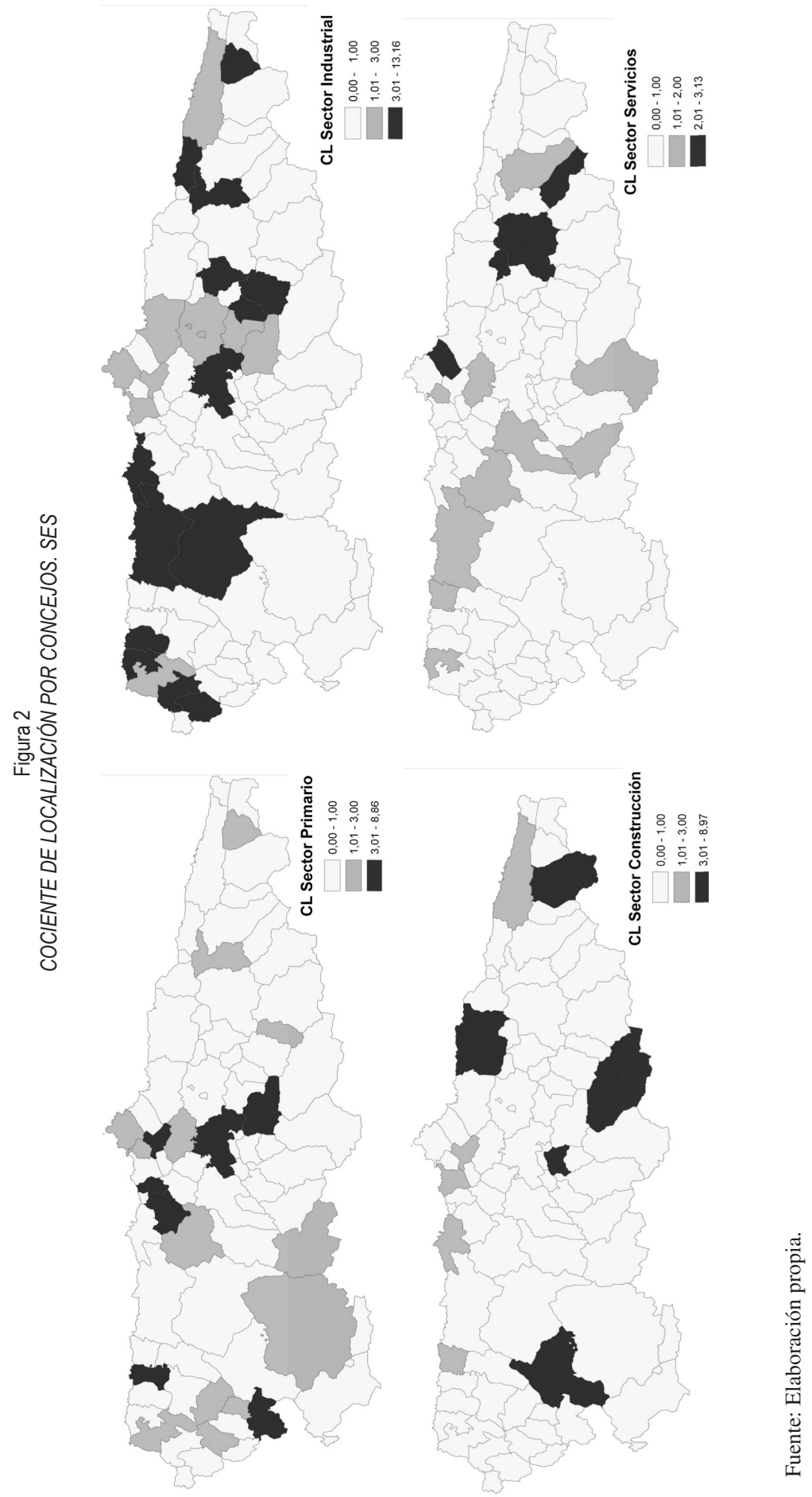


En la Tabla 4 se pueden observar los resultados de ambas pruebas: el valor del índice KMO ha sido de 0,82 que refleja una buena adecuación muestral. De igual forma, en el test de esfericidad de Bartlett se ha obtenido un nivel de significación igual a 0, rechazando la hipótesis nula de que la matriz de correlaciones es igual a una matriz identidad. Por tanto, con estos contrastes también se comprueba la correlación existente entre las variables utilizadas en el análisis y por tanto, se justifica la aplicación del ACP.

Tabla 4

ADECUACIÓN DE LOS DATOS. TEST DE KMO YBARTLETT

Medida de adecuación de la muestra Kaiser-Meyer-Olkin

Test de esfericidad de Bartlett

Aprox. Chi-Cuadrado

1337,871

g1

66

Sig.

, 000

Fuente: Elaboración propia.

Para determinar el número de componentes a extraer se aplica el criteriode Kaiser, el cual recomienda la extracción de aquellos componentes cuyos autovalores son superiores a la unidad.

Tabla 5

CAPACIDAD EXPLICATIVA DE LOS FACTORES

\begin{tabular}{|r|c|c|c|}
\hline \multirow{2}{*}{ Componente } & \multicolumn{3}{|c|}{ Autovalores Iniciales } \\
\cline { 2 - 4 } & $\left(\lambda_{\mathrm{i}}\right)$ & $\begin{array}{c}\text { \% de la } \\
\text { varianza }\end{array}$ & $\begin{array}{c}\text { \% acumulado de } \\
\text { la varianza }\end{array}$ \\
\hline 1 & 9,944 & 82,871 & 82,871 \\
\hline 2 & 1,507 & 12,561 & 95,431 \\
\hline 3 &, 300 & 2,504 & 97,935 \\
\hline 4 &, 137 & 1,138 & 99,073 \\
\hline 5 &, 036 &, 302 & 99,375 \\
\hline 6 &, 030 &, 250 & 99,625 \\
\hline 7 &, 025 &, 206 & 99,831 \\
\hline 8 &, 013 &, 108 & 99,939 \\
\hline 9 &, 006 &, 050 & 99,988 \\
\hline 10 &, 001 &, 010 & 99,998 \\
\hline 11 &, 000 &, 002 & 100,000 \\
\hline 12 & $6,992 \mathrm{E}-6$ & $5,827 \mathrm{E}-5$ & 100,000 \\
\hline
\end{tabular}

Fuente: Elaboración propia. 
A partir de los resultados que se muestran en la Tabla 5, y teniendo en cuenta la regla de Kaiser se han extraído dos componentes, que explican el $95.43 \%$ de la varianza total .

Una vez determinado el número de componentes principales, se calculan las comunalidades, es decir, la proporción de varianza de cada variable, explicada por el conjunto de los factores. El rango de este indicador está entre 0 y 1, por lo tanto, un valor igual a 1 implica que una variable está completamente explicada por el conjunto de componentes.

Tabla 6

REPRESENTATIVIDAD DE LAS VARIABLES A PARTIR DE LOS FACTORES RETENIDOS

\begin{tabular}{|l|r|}
\hline \multicolumn{1}{|c|}{ Variables } & Extracción \\
\hline Empleo &, 986 \\
\hline CI &, 995 \\
\hline RA &, 975 \\
\hline RPpag &, 962 \\
\hline PS &, 965 \\
\hline FBKF &, 907 \\
\hline CK &, 842 \\
\hline Pn &, 995 \\
\hline Rprec &, 954 \\
\hline OTCrec &, 932 \\
\hline Sector2 &, 953 \\
\hline Sector3 &, 985 \\
\hline
\end{tabular}

Fuente: Elaboración propia.

En la Tabla 6 se presentan las comunalidades de cada una de las variables empleadas en el estudio, como se puede apreciar todas son cercanas a 1, con lo cual se puede concluir que el conjunto de variables originales está bien explicado por las componentes extraídas. Entre las magnitudes que presentan una mayor comunalidad destacan el consumo intermedio y la producción, ambos con un 0,995.

Una vez determinado el número de componentes principales, se realiza el análisis de la matriz factorial. Con el objetivo de facilitar la interpretación de las componentes se ha efectuado la rotación Varimax. Como se puede apreciar en la Tabla 7, la primera componente se encuentra directamente relacionada con las variables referidas a los sectores secundario (Sector2) y terciario (Sector3), el nivel de empleo, remuneración asalariados (RA) y prestaciones sociales (PS), además de otras variables económicas como la FBKF, rentas de la propiedad pagadas (RPpag), otras transferencia corrientes recibidas (OTCrec) y el consumo de capital (CK). Mientras que el segundo factor se relaciona positivamente con las variables referidas, en la contabilidad nacional, a las cuentas de producción: consumos intermedios (CI) y producción (Pn), además de las rentas de la propiedad recibidas (RPrec). 
Tabla 7

INTERPRETACIÓN DE LOS FACTORES

\begin{tabular}{|l|c|c|}
\hline \multirow{2}{*}{ Variables } & \multicolumn{2}{|c|}{ Componentes SES } \\
\cline { 2 - 3 } & $\mathbf{1}$ & $\mathbf{2}$ \\
\hline Sector3 &, 983 &, 137 \\
\hline Sector2 &, 960 &, 177 \\
\hline Empleo &, 930 &, 348 \\
\hline FBKF &, 874 &, 378 \\
\hline RA &, 855 &, 493 \\
\hline RPpag &, 850 &, 489 \\
\hline PS &, 811 &, 555 \\
\hline OTCrec &, 800 &, 540 \\
\hline CK &, 782 &, 481 \\
\hline CI &, 203 &, 977 \\
\hline Pn &, 303 &, 950 \\
\hline Rprec &, 466 &, 858 \\
\hline
\end{tabular}

Rotación: Varimax con normalización de Kaiser.

Fuente: Elaboración propia.

Por último, se ha efectuado una clasificación de los concejos de acuerdo con los factores retenidos mediante un análisis gráfico de las puntuaciones factoriales rotadas como se muestra en la Figura 3. Así, podemos distinguir un primer grupo de municipios, constituido por Gijón y Siero, que presentan mayor dinamismo, ya que tienen un peso importante en todas las variables consideradas (Factores 1 y 2). Un segundo grupo estaría formado por aquellos concejos que se caracterizan por tener un mayor peso en las variables propiamente económicas (factor 1) y menor en las de producción (Factor 2), como son Oviedo, Avilés, Tineo y Cangas de Narcea. Un tercer grupo estaría constituido por Llanera y Valdés, que pese a contar con una baja representación de las variables asociadas al primer componente (como el nivel de empleo, RA y PS), reflejan unos elevados niveles de producción y consumos intermedios (Factor 2). El resto de municipios muestran un menor peso tanto en las variables propiamente económicas como en las de producción (Factores 1 y 2).

A continuación se efectúa un análisis del SES, partiendo de la consideración separada de los dos tipos de entidades más relevantes: las cooperativas y las sociedades laborales.

A partir de la Tabla 8 se puede concluir la existencia de correlación entre las variables analizadas, al concluir con el rechazo del test de esfericidad de Bartlett; además, el resultado de la medida de adecuación de la muestra KMO para los dos tipos societarios analizados es elevada, justificándose la utilización del método de ACP. 
Figura 1

CLASIFICACIÓN DE LOS CONCEJOS. SES

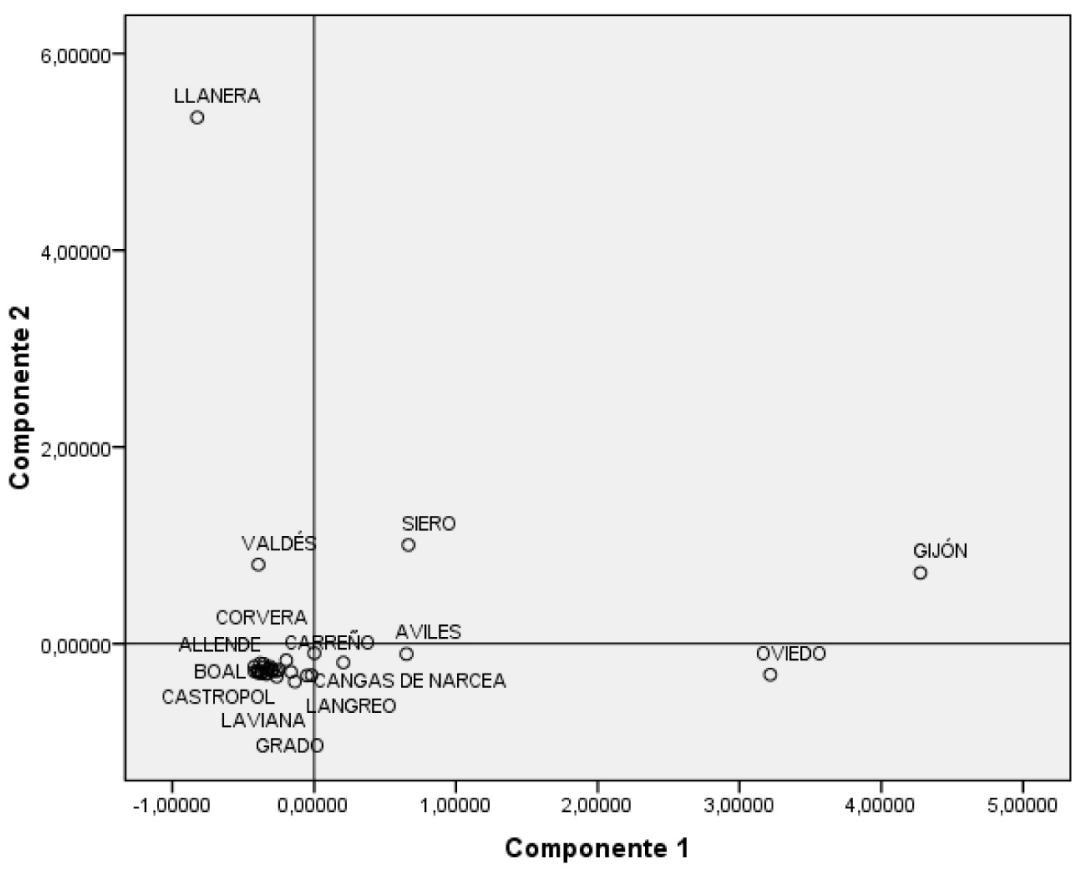

Fuente: Elaboración propia.

Tabla 8

ADECUACIÓN DE LOS DATOS. TEST DE KMO YBARTLETT

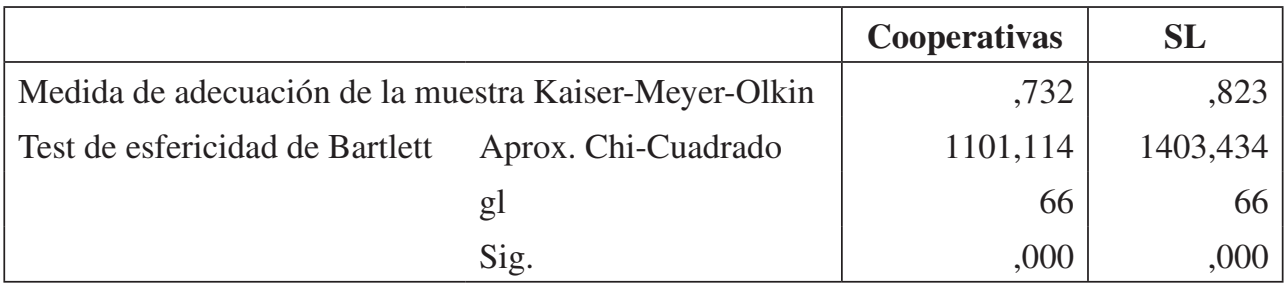

Fuente: Elaboración propia.

Como se muestra en la Tabla 9 para poder efectuar una comparación directa con el SES, se han extraído dos componentes, los cuales explican el 89,38\% de la varianza en las cooperativas y del $96,61 \%$ en las sociedades laborales. Se puede observar el mayor porcentaje de varianza total explicada presentada en el primer componente de las sociedades laborales (94\%) frente al $71,91 \%$ en las cooperativas. 
Tabla 9

CAPACIDAD EXPLICATIVA DE LOS FACTORES SEGÚN TIPOLOGÍA

\begin{tabular}{|c|c|c|c|c|c|c|}
\hline \multirow{2}{*}{ Componente } & \multicolumn{3}{|c|}{$\begin{array}{l}\text { Autovalores iniciales } \\
\text { Cooperativas }\end{array}$} & \multicolumn{3}{|c|}{$\begin{array}{c}\text { Autovalores iniciales } \\
\text { SL }\end{array}$} \\
\hline & Total & $\begin{array}{c}\% \text { de } \\
\text { varianza }\end{array}$ & $\begin{array}{r}\% \text { acumulado } \\
\text { de la varianza }\end{array}$ & Total & $\begin{array}{c}\% \text { de } \\
\text { varianza }\end{array}$ & $\begin{array}{r}\% \text { acumulado } \\
\text { de la varianza }\end{array}$ \\
\hline 1 & 8,629 & 71,905 & 71,905 & 11,280 & 93,997 & 93,997 \\
\hline 2 & 2,096 & 17,471 & 89,376 & ,313 & 2,611 & 96,608 \\
\hline 3 & ,457 & 3,809 & 93,185 & , 187 & 1,562 & 98,170 \\
\hline 4 &, 348 & 2,901 & 96,086 & , 105 & ,874 & 99,044 \\
\hline 5 & ,261 & 2,174 & 98,260 & ,066 & ,553 & 99,597 \\
\hline 6 & , 123 & 1,024 & 99,284 & ,025 & ,212 & 99,809 \\
\hline 7 & ,047 & ,393 & 99,677 & ,010 & ,087 & 99,896 \\
\hline 8 & ,026 & ,219 & 99,895 & ,008 & ,065 & 99,961 \\
\hline 9 & ,009 & 074 & 99,969 & ,002 & ,021 & 99,981 \\
\hline 10 & ,003 & ,027 & 99,996 & ,002 & ,017 & 99,998 \\
\hline 11 & ,001 & ,004 & 100,000 &, 000 & ,001 & 100,000 \\
\hline 12 & $6,26 \mathrm{E}-6$ & $5,224 \mathrm{E}-5$ & 100,000 & $3,090 \mathrm{E}-5$ & ,000 & 100,000 \\
\hline
\end{tabular}

Fuente: Elaboración propia.

Las comunalidades (Tabla 10) muestran valores próximos a la unidad para todas las variables y tipos de entidad analizadas. En lo que se refiere a las cooperativas, destacan la remuneración a asalariados, las prestaciones sociales y la producción como las variables que explican una mayor proporción de la varianza. En cuanto a las sociedades laborales, todos los valores son superiores a 0,92 , destacando las variables de producción, consumo intermedio y rentas de la propiedad recibida.

La definición de las componentes una vez aplicada la rotación (Tabla 11) muestra en el caso de las cooperativas, un primer factor formado por variables exclusivamente económicas; la segunda componente está constituida por magnitudes referentes al nivel de empleo y a la participación en los sectores económicos. Por otra parte, en el caso de las sociedades laborales, se muestra que la primera componente presenta una elevada relación con las variables referentes al nivel de empleo, sectores económicos y la mayoría de las variables económicas a excepción de la renta de la propiedad recibida, consumo intermedio y producción.

Finalmente, el análisis gráfico de las puntuaciones factoriales representadas en la Figura 4, muestra para las cooperativas que en los concejos de Gijón, Siero, Avilés y Valdés resalta la importancia de las variables recogidas en los dos factores considerados. Por otro lado, Cangas de Narcea, El Franco y Llanera muestran un mayor peso de las variables que hemos denominado económicas y menor del empleo, así como menor número de empresas en los sectores de actividad secundario y terciario (Factor 2); este comportamiento es el opuesto 
Tabla 10

REPRESENTATIVIDAD DE LAS VARIABLES. COOPERATIVAS Y SL

\begin{tabular}{|l|r|r|}
\hline \multirow{2}{*}{ Variables } & \multicolumn{2}{|c|}{ Extracción } \\
\cline { 2 - 3 } & Cooperativas & \multicolumn{1}{c|}{ SL } \\
\hline Empleo &, 898 &, 938 \\
\hline CI &, 963 &, 989 \\
\hline RA &, 985 &, 972 \\
\hline RPpag &, 910 &, 981 \\
\hline PS &, 985 &, 970 \\
\hline FBKF &, 835 &, 943 \\
\hline CK &, 688 &, 972 \\
\hline Pn &, 966 &, 996 \\
\hline RPrec &, 929 &, 982 \\
\hline OTCrec &, 845 &, 950 \\
\hline Sector2 &, 821 &, 929 \\
\hline Sector3 &, 901 &, 970 \\
\hline
\end{tabular}

Fuente: Elaboración propia.

Tabla 11

INTERPRETACIÓN DE LOS FACTORES. COOPERATIVAS Y SL

\begin{tabular}{|c|c|c|c|c|c|}
\hline \multirow{2}{*}{ Variables } & \multicolumn{2}{|c|}{$\begin{array}{l}\text { Componentes } \\
\text { Cooperativas }\end{array}$} & \multirow{2}{*}{ Variables } & \multicolumn{2}{|c|}{ Componentes SL } \\
\hline & 1 & 2 & & 1 & 2 \\
\hline $\mathrm{CI}$ & ,977 & ,089 & CK & ,897 & ,409 \\
\hline Pn & ,976 & ,115 & Sector3 & ,844 & ,507 \\
\hline Rprec & ,964 &,- 016 & Sector2 & ,762 & ,591 \\
\hline OTCrec & ,846 & ,359 & OTCrec &, 741 & ,633 \\
\hline PS &, 831 &, 542 & RPpag & ,735 & 663 \\
\hline FBKF & ,793 & ,454 & FBKF &, 711 & ,661 \\
\hline RA & ,793 & ,597 & PS & ,703 & ,690 \\
\hline $\mathrm{CK}$ & ,768 & ,314 & $\mathrm{RA}$ &, 703 & ,692 \\
\hline RPpag & ,759 &, 577 & Empleo & ,696 & ,674 \\
\hline Sector3 &, 163 & ,935 & Rprec & ,402 & ,906 \\
\hline Empleo & ,262 & ,911 & $\mathrm{CI}$ & ,666 & ,738 \\
\hline Sector2 &, 114 & ,899 & Pn & ,685 &, 726 \\
\hline
\end{tabular}

Método de Rotación: Varimax con normalización de Kaiser. Fuente: Elaboración propia. 
al que se muestra en Oviedo y Tineo, en los cuales están ubicadas un número importante de empresas de dichos sectores, con un alto nivel de empleo y menos peso de las variables económicas. Por su parte, en las sociedades laborales, los concejos de Gijón y Llanera presentan una elevada participación de las variables que definen ambos componentes. Además, Oviedo, Avilés, Mieres, Langreo y Laviana son concejos en los que las empresas ubicadas en ellos presentan una elevada participación en las variables estrictamente económicas, pero menor peso de las relacionadas con la producción. Por otra parte, los concejos de Somiedo y Tapia de Casariego muestran una menor participación en el conjunto de variables económicas señaladas y un elevado peso en las magnitudes referidas a la producción.

CLASIFICACIÓN DE LOS CONCEJOS. COOPERATIVAS Y SL
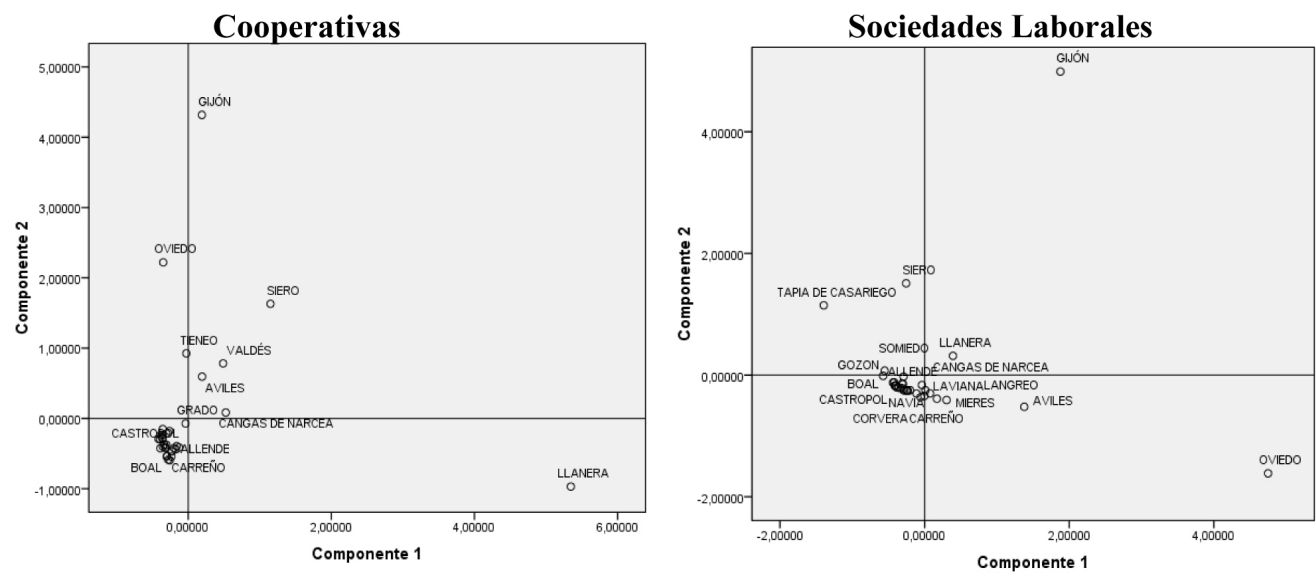

Fuente: Elaboración propia.

\subsection{Caracterización de los concejos: un resumen}

A modo de conclusión de los resultados anteriormente expuestos, se ha realizado una tabla en la que se recogen los valores de los indicadores determinados y se caracteriza a los concejos considerados.

En el cuadro que sigue se han distinguido la Economía Social en su conjunto, las cooperativas y las Sociedades Laborales. Para cada tipo de entidad se ha recogido el factor o componente principal que es relevante; si en el concejo analizado no es relevante ninguno de los factores extraídos se deja un espacio vacío. Además se señala si el concejo está especializado en alguna actividad (Sí/No) y, por último, se muestra si alguna de las actividades económicas señaladas ( $\mathrm{AB}, \mathrm{CD}, \mathrm{F}, \mathrm{G}$ a $\mathrm{O})$ se encuentra localizada en el territorio considerado.

\section{CONCLUSIONES}

La economía social es un sector relevante para la economía de un país o una región, sin embargo, la información disponible sobre dicho sector aún es escasa. Por otra parte, consi- 
Tabla 12

CARACTERIZACIÓN DE LOS CONCEJOS DE ACUERDO A LOS INDICADORES DETERMINADOS

\begin{tabular}{|c|c|c|c|c|c|c|c|c|c|}
\hline & & SES & & & Coop & & & SL & \\
\hline & $\begin{array}{c}\text { Factor } \\
\text { relevante }\end{array}$ & $\mathrm{CE}_{\mathrm{r}}$ & $\mathbf{L}_{\mathrm{sr}}$ & $\begin{array}{c}\text { Factor } \\
\text { relevante }\end{array}$ & $\mathrm{CE}_{\mathrm{r}}$ & $\mathbf{L}_{\mathrm{sr}}$ & $\begin{array}{c}\text { Factor } \\
\text { relevante }\end{array}$ & $\mathrm{CE}_{\mathrm{r}}$ & $\mathbf{L}_{\mathrm{sr}}$ \\
\hline ALLANDE & & $\mathrm{Si}$ & $\mathrm{F}$ & & No & & & Sí & $\mathrm{F}$ \\
\hline AVILES & F1 & No & $\mathrm{AB}, \mathrm{G}$ a O & $\mathrm{F} 1, \mathrm{~F} 2$ & No & $\mathrm{A}, \mathrm{B}$ & F1 & No & $\mathrm{GaO}$ \\
\hline $\begin{array}{ll}\text { CANGAS } & \text { DE } \\
\text { NARCEA } & \\
\end{array}$ & $\mathrm{F} 1$ & No & $\mathrm{AB}$ & $\mathrm{F} 1, \mathrm{~F} 2$ & No & $\mathrm{A}, \mathrm{B}$ & & No & $\mathrm{GaO}$ \\
\hline CARREÑO & & $\mathrm{Si}$ & $\mathrm{GaO}$ & & Sí & $\mathrm{A}, \mathrm{B}$ & F1 & No & $\mathrm{GaO}$ \\
\hline CASTROPOL & & No & $\mathrm{GaO}$ & & Sí & C,D & & Sí & $\mathrm{GaO}$ \\
\hline CORVERA & & No & $\mathrm{AB}, \mathrm{F}$ & & Sí & $\mathrm{A}, \mathrm{B}$ & & No & $\mathrm{F}$ \\
\hline CUDILLERO & & $\mathrm{Si}$ & $\mathrm{C}, \mathrm{D}, \mathrm{F}$ & & Sí & $\mathrm{F}$ & & Sí & $\mathrm{C}, \mathrm{D}$ \\
\hline EL FRANCO & & $\mathrm{Si}$ & $\mathrm{C}, \mathrm{D}$ & & No & & & Sí & $\mathrm{CD}, \mathrm{G}$ a O \\
\hline GIJÓN & $\mathrm{F} 1, \mathrm{~F} 2$ & No & $\mathrm{F}, \mathrm{G}$ a O & $\mathrm{F} 1, \mathrm{~F} 2$ & No & & $\mathrm{F} 1, \mathrm{~F} 2$ & No & $\mathrm{F}$ \\
\hline GOZON & & $\mathrm{Si}$ & & & Sí & $\mathrm{C}, \mathrm{D}$ & & Sí & $\mathrm{GaO}$ \\
\hline GRADO & & No & $\mathrm{GaO}$ & & No & & & No & C,D \\
\hline LANGREO & & No & & & Sí & $\mathrm{C}, \mathrm{D}$ & $\mathrm{F} 1$ & No & \\
\hline LAVIANA & & $\mathrm{Si}$ & C,D & & Sí & $\mathrm{F}$ & F1 & Sí & C,D \\
\hline LENA & & No & $\mathrm{GaO}$ & & No & & & No & $\mathrm{GaO}$ \\
\hline LLANERA & $\mathrm{F} 2$ & No & $\mathrm{AB}, \mathrm{GaO}$ & F1 & No & C,D & $\mathrm{F} 1, \mathrm{~F} 2$ & No & $\mathrm{F}, \mathrm{G}$ a O \\
\hline MIERES & & No & $\mathrm{AB}$ & & No & $\mathrm{A}, \mathrm{B}$ & $\mathrm{F} 1$ & No & $\mathrm{GaO}$ \\
\hline NAVA & & $\mathrm{Si}$ & C,D & & No & $\mathrm{A}, \mathrm{B}$ & & Sí & \\
\hline NAVIA & & No & $\mathrm{F}, \mathrm{GaO}$ & & Sí & C,D & & Sí & $\mathrm{C}, \mathrm{D}$ \\
\hline NOREÑA & & $\mathrm{Si}$ & & & No & $F$ & & No & $\mathrm{GaO}$ \\
\hline OVIEDO & $\mathrm{F} 1$ & No & $\mathrm{A}, \mathrm{B}, \mathrm{C}, \mathrm{D}, \mathrm{F}$ & $\mathrm{F} 2$ & Sí & C,D & $\mathrm{F} 1$ & No & \\
\hline PARRES & & No & C.D & & No & $\mathrm{A}, \mathrm{B}$ & & No & $\mathrm{F}$ \\
\hline $\begin{array}{l}\text { PEÑAMELLERA } \\
\text { ALTA }\end{array}$ & & $\mathrm{Si}$ & $\mathrm{A}, \mathrm{B}, \mathrm{C}, \mathrm{D}$ & & No & C,D & & No & $\mathrm{GaO}$ \\
\hline PILOÑA & & $\mathrm{Si}$ & $\mathrm{G} \mathrm{a} \mathrm{O}$ & & Sí & & & Sí & $\mathrm{C}, \mathrm{D}$ \\
\hline PRAVIA & & No & $\mathrm{AB}, \mathrm{G}$ a O & & Sí & $\mathrm{GaO}$ & & Sí & $\mathrm{GaO}$ \\
\hline RIBADESELLA & & $\mathrm{Si}$ & C,D & & Sí & $\mathrm{A}, \mathrm{B}$ & & No & \\
\hline SALAS & & No & $\mathrm{GaO}$ & & Sí & C,D & & Sí & C,D \\
\hline $\begin{array}{l}\text { SAN MARTIN DEL } \\
\text { REY AURELIO }\end{array}$ & & $\mathrm{Si}$ & C,D & & No & & & No & \\
\hline SIERO & $\mathrm{F} 1, \mathrm{~F} 2$ & No & & $\mathrm{F} 1, \mathrm{~F} 2$ & Sí & $\mathrm{C}, \mathrm{D}$ & $\mathrm{F} 1$ & Sí & $\mathrm{C}, \mathrm{D}$ \\
\hline SOMIEDO & & $\mathrm{Si}$ & $\mathrm{AB}$ & & No & C,D & F2 & No & \\
\hline $\begin{array}{l}\text { TAPIA DE CASA- } \\
\text { RIEGO }\end{array}$ & & $\mathrm{Si}$ & $\mathrm{C}, \mathrm{D}, \mathrm{G}$ a O & & No & & F2 & No & $\mathrm{A}, \mathrm{B}$ \\
\hline TINEO & $\mathrm{F} 2$ & $\mathrm{Si}$ & $\mathrm{C}, \mathrm{D}$ & $\mathrm{F} 2$ & No & $\mathrm{C}, \mathrm{D}$ & & Sí & $\mathrm{GaO}$ \\
\hline VALDÉS & F1 & No & $\mathrm{C}, \mathrm{D}, \mathrm{G}$ a O & $\mathrm{F} 1, \mathrm{~F} 2$ & Sí & C,D & & Sí & $\mathrm{GaO}$ \\
\hline VEGADEO & & $\mathrm{Si}$ & $\mathrm{C}, \mathrm{D}$ & & Sí & C,D & & Sí & C,D \\
\hline
\end{tabular}

Fuente: elaboración propia. 
deramos que la óptica espacial tiene una especial relevancia en los estudios económicos, ya que proporciona una gran riqueza a los resultados obtenidos. Por todo ello, en este artículo se realiza un análisis en el que a partir de esta perspectiva se han caracterizado a los concejos de la comunidad asturiana.

Inicialmente se analiza la existencia de una relación entre la actividad llevada a cabo por la empresa y su ubicación, mediante la realización de un análisis de la varianza, el cual muestra que la actividad en la que se encuadre una empresa del sector de la economía social (SES) está influida por su situación geográfica. Sin embargo, al efectuar este análisis individualizado sobre las cooperativas y las sociedades laborales, se deriva que no existe tal influencia. Este resultado contradictorio puede deberse a que dentro del sector de la economía social se incluyen las cofradías de pescadores y las sociedades agrarias de transformación, las cuales dado su especial carácter «sesgan» los resultados.

Posteriormente, se ha analizado la especialización de los propios concejos en ciertos sectores y sobre la localización de las actividades productivas en los mismos. Por lo que se refiere al conjunto de la Economía Social, el análisis del cociente de localización refleja la importancia de los concejos de Corvera de Asturias, Mieres, Oviedo, Soto del Barco y Coaña en el sector primario; los municipios de Vegadeo, Peñameñera Alta, El Franco, y Ribadesella en el sector industrial; los concejos de Morcín, Allande, Aller, Cabrales y Villaviciosa, en el sector de la construcción; y, por último, Amieva, Cabranes, Piloña y Carreño en el sector servicios.

Los concejos de Morcín, Aller, Taramundi, Muros de Nalón, El Franco y Nava presentan un mayor grado de especialización, junto con Gijón, Oviedo, Avilés y Valdés.

Dado que se dispone de información económica bastante variada procedente de las Cuentas Satélite de la Economía Social Asturiana, y mediante la utilización de la técnica multivariante de análisis de componentes principales, se ha resumido dicha información en un número menor de variables que contiene una buena parte de la información inicial sobre el comportamiento del SES en cada uno de los concejos asturianos. Así, tanto para el conjunto de la economía social, como para las cooperativas y sociedades laborales, por separado, se han seleccionado dos componentes principales. En relación a la interpretación de los factores retenidos, en el caso del SES, el primer componente está compuesto principalmente por las variables referentes a los sectores de actividad de servicios y secundario, el nivel de empleo y algunas variables económicas relacionada con este último, como la remuneración de asalariados y las prestaciones sociales. Por otra parte, el segundo componente se relaciona con las variables económicas pertenecientes a la cuenta de producción (consumo intermedio y producción). Las sociedades cooperativas, presentan una distribución bien definida, en el primer componente se sitúan todas las variables económicas, mientras que en el segundo, se ubican las variables relacionadas con el sector de actividad secundario y terciario. Por último, en las sociedades laborales, se muestra un comportamiento similar al del SES en su conjunto.

El análisis gráfico de las puntuaciones factoriales rotadas refleja la importancia del concejo de Gijón, que en los tres tipos de entidades consideradas muestra una elevada participación de las variables relacionadas con ambos componentes. También destacan en el conjunto de la Economía Social, los concejos de Siero, Oviedo y Llanera; en las cooperativas también sobresalen los concejos de Siero, Llanera y Oviedo; y en las sociedades laborales además de Gijón y Siero destacan, aunque en una menor medida, Avilés, Tapia de Casariego y Llanera. 
Finalmente, y de acuerdo al conjunto de los indicadores anteriormente determinados, se caracteriza a los municipios asturianos.

\section{REFERENCIAS BIBLIOGRÁFICAS}

ANGOA, I., PÉREZ-MENDOZA, S. y POLÈSE M. (2009): «Los tres Méxicos: análisis de la distribución espacial del empleo en la industria y los servicios superiores, por tamaño urbano y por región» en Eure, núm. 35, vol. 104, 121-144, abril 2009. Disponible en http://www.eure.cl/numero/los-tres-mexicos-analisis-de-la-distribucion-espacial-del-empleo-en-la-industria-y-los-servicios-superiores-por-tamano-urbano-y-por-region/

ALONSO, O., CHAMORRO, J. M. y GONZÁLEZ, X. (2004): «Agglomeration economies in manufacturing industries: the case of Spain». Applied Economics, núm. 36, vol. 8, 103-116.

BARTLETT, M.S. (1954): «A note of the multiplying factors for various chi square approximations». Journal of the Royal Statistical Society B, núm. 16, 296-298.

BEYERS, W. (1991): «Trends in the producer services in the USA: the last decade», en DANIELS, P. W., coord. Services and metropolitan development, Edit. Routledge, 146-172.

BEYERS, W. y LINDAHL, D. (1996): «Croissance et localisation des services aux entreprises aux Etats Unis». L’Espace Géographique, núm. 4, 315-322.

COFFEY, W. y SHEARMOUR, R. (1997): «The growth and Location of High Order Services in the Canadian Urban System, 1971-1991», Professional Geographer, núm. 49, vol. 4, 404-418.

DÁVILA, A. (2004): «México: Concentración y localización del empleo manufacturero, 1980-1998». Economía Mexicana, núm. XIII, vol. 2, 209-254.

DESMET, K. y FAFCHAMPS, M. (2005): «Changes in the spatial concentration of employment across US counties: a sectoral análisis (1972-2000)». Journal of Economic Geography, núm. 5, 261-284.

GAIGNÉ, C., PIGUET, V. y SCMITT, B. (2005): «Évolution récente de l'emploi industriel dans les pays territoires ruraux et urbains: une analyse structurelle-géographique sur des données françaises». Revue d'Économie Régionale et Urbaine, núm. 1, 3-30.

HENDERSON, V. (1997): «Medium sized cities». Regional Science and Urban Economics, núm. 27, 583-612.

HENDERSON, V. (2003): «Marshall's scale economies». Journal of Urban Economics, núm. 53, 1-28.

HURTADO, A., RAMOS, C. Y FERNÁNDEZ, E. (2012): «Elaboración de las Cuentas Satélite de las entidades de la Economía Social. Un estudio de caso para la Economía Asturiana en el año 2005» Ciriec-España, núm. 74, 5-37.

INGRAM, G. K. (1998): «Patterns of metropolitan development: what have we learned?» Urban Studies, núm. 35, vol. 7, 109-1035.

KAISER, H. F. (1974): «An index of factorial simplicity». Psychometrika, núm. 39, 31-36.

LEÓN, G. (2008): Análisis de Encuesta mediante Técnicas de Reducción de Datos. Caso: Caracterización de la Percepción Pública de la Ciencia y la Biotecnología en Mérida. Tesis para obtener el grado de Magíster Scientiae en Estadística. Mérida, Venezuela, Universidad de Los Andes. 
LEY 5/2011, de 29 de marzo, de Economía Social. BOE de 30 de marzo de 2011.

LEMELIN A y POLÈSE M., (1993): «The location of employment in developing countries. Models of urbanization and comparative analyses of the Canadian and Mexican urban systems». Estudios Demográficos y Urbanos. Núm. 8, vol. 2, 331-60.

MONZÓN, J.L., ANATUÑANO, I. y MARCO F. (2010): Sectores de la nueva economía 20+20. Economía Social. Fundación EOI. Madrid.

MTIN (2005): Estadísticas de Economía Social, Dirección General de Fomento de la Economía Social y del Fondo Social Europeo, Ministerio de Trabajo y Asuntos Sociales, España.

OLKIN, I., GHURYE, S. G., HOEFFDING, W., MADOW, W. G., y MANN, H. B. (1960): Contributions to Probability and Statistics: Essays in Honor of Harold Hotelling. Stanford University Press, Stanford, CA.

POLÈSE, M. (1994): Economie urbaine et régionale: logique spatiale des mutations économiques, Paris.

POLĖSE, M. y SHEARMUR, R. (2004): «Is distance really dead? Comparing industrial location patterns over time in Canada». International Regional Science Review, núm. 27, vol. 4, 431-457.

POLÈSE, M. y SHEARMUR, R. (2006): «Growth and location of economic activity: the spatial dynamics of industries in Canada (1971-2001)». Growth and Change, núm. 37, vol. 3, 362-395.

RUBIERA, F. (2005): Los servicios avanzados a las empresas. Dinámicas de localización, patrones de externalización y efectos sobre el desarrollo regional. España: Civitas.

TERRAL L. y POLÈSE, M. (2006): «À la recherche de grandes régularités dans la localisation de l'activité économique. Analyse du tertiaire supérieur et de l'industrie manufacturière pour l'espace nord-américain». Revue canadienne de sciences régionales, núm. 29, vol. 2, 275-298. 
Figura A1

MAPA DEL PRINCIPADO DE ASTURIAS POR CONCEJOS Y COMARCAS ${ }^{11}$

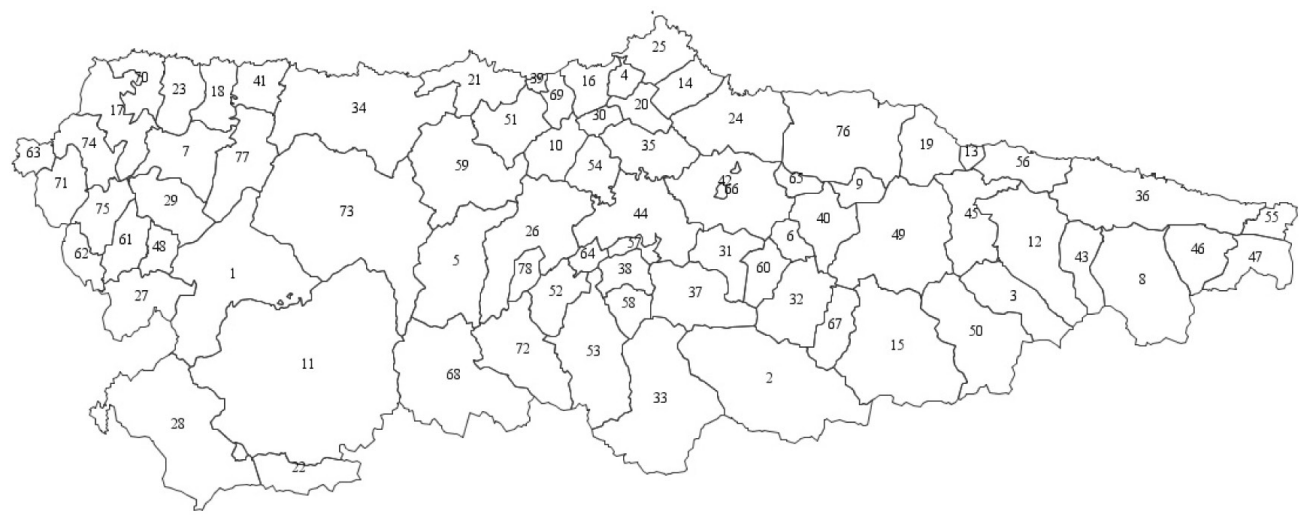

Tabla A1

DIVISIÓN GEOGRÁFICA DEL PRINCIPADO DE ASTURIAS POR CONCEJOS Y COMARCAS

\begin{tabular}{|c|c|c|c|c|}
\hline Allande - C6 & 27 & Grandas de Salime - C3 & 53 & Quirós - C8 \\
\hline Aller - C2 & 28 & Ibias - C6 & 54 & Regueras, Las - C8 \\
\hline Amieva - C7 & 29 & Illano - C3 & 55 & Ribadedeva - C7 \\
\hline Avilés - C1 & 30 & Illas - C1 & 56 & Ribadesella - C7 \\
\hline Belmonte de Miranda - C8 & 31 & Langreo - C5 & 57 & Ribera de Arriba - C8 \\
\hline Bimenes - C8 & 32 & Laviana - C5 & 58 & Riosa - C8 \\
\hline Boal - C3 & 33 & Lena - C2 & 59 & Salas - C8 \\
\hline Cabrales - C7 & 34 & Valdés - C3 & 60 & S. M. del Rey Aurelio - C5 \\
\hline Cabranes - C8 & 35 & Llanera - C8 & 61 & San Martín de Oscos - C3 \\
\hline Candamo - C1 & 36 & Llanes - C7 & 62 & Santa Eulalia de Oscos - C3 \\
\hline Cangas del Narcea - C6 & 37 & Mieres - C2 & 63 & San Tirso de Abres - C3 \\
\hline Cangas de Onís - C7 & 38 & Morcín - C8 & 64 & Santo Adriano - C8 \\
\hline Caravia - C7 & 39 & Muros de Nalón - C1 & 65 & Sariego - C8 \\
\hline Carreño - C4 & 40 & Nava - C8 & 66 & Siero - C8 \\
\hline Caso - C5 & 41 & Navia - C3 & 67 & Sobrescobio - C5 \\
\hline Castrillón - C1 & 42 & Noreña - C8 & 68 & Somiedo - C8 \\
\hline Castropol - C3 & 43 & Onís - C7 & 69 & Soto del Barco - C1 \\
\hline Coaña - C3 & 44 & Oviedo - C8 & 70 & Tapia de Casariego - C3 \\
\hline Colunga - C7 & 45 & Parres - C7 & 71 & Taramundi - C3 \\
\hline Corvera de Asturias - C1 & 46 & Peñamellera Alta - C7 & 72 & Teverga - C8 \\
\hline Cudillero - C1 & 47 & Peñamellera Baja - C7 & 73 & Tineo - C6 \\
\hline Degaña - C6 & 48 & Pesoz - C3 & 74 & Vegadeo - C3 \\
\hline Franco, $\mathrm{El}-\mathrm{C} 3$ & 49 & Piloña - C7 & 75 & Villanueva de Oscos - C3 \\
\hline Gijón - C4 & 50 & Ponga - C7 & 76 & Villaviciosa - C4 \\
\hline Gozón-C1 & 51 & Pravia-C1 & 77 & Villayón - C3 \\
\hline Grado - C8 & 52 & Proaza - C8 & 78 & Yernes y Tameza - C8 \\
\hline
\end{tabular}

11 Las comarcas se especifican justo después del nombre del concejo, con la letra $\mathrm{C}$ y el número correspondiente a la misma. 
Tabla A.2.

COCIENTE DE LOCALIZACIÓN SEGÚN TIPO DE ENTIDAD DEL SES

\begin{tabular}{|c|c|c|c|c|c|c|c|c|c|c|c|c|}
\hline \multirow{2}{*}{ Concejos } & \multicolumn{4}{|c|}{ SES } & \multicolumn{4}{|c|}{ Cooperativas } & \multicolumn{4}{|c|}{ SL } \\
\hline & $\mathbf{A , B}$ & C,D & F & Ga 0 & $\overline{A, B}$ & C,D & $\mathrm{F}$ & $\mathrm{GaO}$ & $\overline{A, B}$ & C,D & $F$ & $\mathrm{GaO}$ \\
\hline Allande & 0,72 & 0,00 & 7.96 & 0,18 & 1.56 & 0.00 & 0.00 & 0.39 & 0,00 & 0,00 & 14.75 & 0,00 \\
\hline Aller & 0,00 & 0,00 & 7,36 & 0,00 & 0,00 & 0,00 & 0,00 & 0,00 & 0,00 & 0,00 & 7,36 & 0,00 \\
\hline Amieva & 0,00 & 0,00 & 0,00 & 3,13 & 0,00 & 0,00 & 0,00 & 3,13 & 0,00 & 0,00 & 0,00 & 0,00 \\
\hline Avilés & 1.56 & 0,60 & 0,15 & 1.34 & 3,25 & 0,00 & 0.31 & 1.40 & 0,00 & 1.39 & 0.00 & 1.21 \\
\hline Cabrales & 0,00 & 0,00 & 4,41 & 0,95 & 0,00 & 0,00 & 0,00 & 0,00 & 0,00 & 0,00 & 4,41 & 0,95 \\
\hline Cabranes & 0,00 & 0,00 & 0,00 & 2,57 & 0,00 & 0,00 & 0,00 & 0,00 & 0,00 & 0,00 & 0,00 & 2,57 \\
\hline Cangas del Narcea & 2,09 & 0.48 & 0,00 & 0,76 & 2.57 & 0,00 & 0,00 & 0.61 & 0,00 & 2.55 & 0,00 & 1.41 \\
\hline Cangas de Onís & 0,86 & 0,00 & 0,00 & 1,29 & 0,00 & 0,00 & 0,00 & 1,50 & 0,00 & 0,00 & 0,00 & 0,00 \\
\hline Carreño & 0,14 & 0,24 & 0,00 & 2,23 & 4,64 & 0,00 & 0,00 & 1,86 & 0,00 & 0,69 & 0,00 & 1,75 \\
\hline Castrillón & 0,00 & 1,24 & 2,02 & 0,73 & 0,00 & 0,00 & 0,00 & 0,00 & 0,00 & 0,00 & 2.79 & 1,01 \\
\hline Castropol & 1,05 & 1,49 & 0,00 & 0,83 & 0,00 & 3,01 & 0,00 & 0,84 & 0,00 & 0,00 & 0,00 & 3,28 \\
\hline Coaña & 4,68 & 0,00 & 0,00 & 0,00 & 0,00 & 0,00 & 0,00 & 0,00 & 0,00 & 0,00 & 0,00 & 0,00 \\
\hline Corvera de Asturias & 8,86 & 1,00 & 1,67 & 0,35 & 15,85 & 1.44 & 0.00 & 0,00 & 0,00 & 0.46 & 3.79 & 0,79 \\
\hline Cudillero & 0,24 & 5,98 & 1,13 & 0,19 & 0,00 & 0,00 & 10,19 & 0,00 & 0,00 & 7,31 & 0,00 & 0,24 \\
\hline Franco, El & 0,00 & 9.45 & 0,00 & 0,19 & 0,00 & 0,00 & 0,00 & 0,00 & 0,00 & 5,14 & 0,00 & 1,17 \\
\hline Giión & 0,00 & 1.62 & 0,75 & 0.91 & 0,00 & 0,11 & 0.38 & 1.33 & 0,00 & 1.07 & 1.36 & 0.94 \\
\hline Gozón & 1,08 & 2,70 & 0,00 & 0,31 & 1,83 & 2,65 & 0,00 & 0,00 & 0,00 & 0,00 & 0,00 & 2,22 \\
\hline Grado & 0,50 & 0,69 & 0,00 & 1,31 & 0,57 & 0,00 & 0,00 & 1,37 & 0,00 & 6,29 & 0,00 & 0,86 \\
\hline Grandas de Salime & 3,09 & 0,00 & 0,00 & 0,00 & 3,09 & 0,00 & 0,00 & 0,00 & 0,00 & 0,00 & 0,00 & 0,00 \\
\hline Illano & 1,80 & 0,00 & 0,00 & 0,00 & 1,80 & 0,00 & 0,00 & 0,00 & 0,00 & 0,00 & 0,00 & 0,00 \\
\hline Langreo & 0,00 & 2,43 & 0,00 & 0,50 & 0,00 & 3,58 & 0,00 & 0,00 & 0,00 & 2,11 & 0,00 & 0,65 \\
\hline Laviana & 0,00 & 6.17 & 0,11 & 0.45 & 0,00 & 0,00 & 7.54 & 0,00 & 0.00 & 5.76 & 0,00 & 0.53 \\
\hline Lena & 0,00 & 0,00 & 0,00 & 1,42 & 0,00 & 0,00 & 0,00 & 0,00 & 0,00 & 0,00 & 0,00 & 1,42 \\
\hline Valdés & 0,26 & 4,39 & 0,15 & 1,24 & 0,09 & 5,22 & 0,00 & 1,28 & 0,00 & 0,00 & 1,61 & 1,73 \\
\hline Llanera & 2,17 & 0,60 & 0,00 & 1.28 & 7,04 & 0.58 & 0.00 & 1,05 & 0,00 & 0.53 & 0,00 & 1.42 \\
\hline Llanes & 0,65 & 1,98 & 1,75 & 0,82 & 0,00 & 0,00 & 0,00 & 0,00 & 0,00 & 2,19 & 1,93 & 0,91 \\
\hline Mieres & 6,73 & 1,73 & 0,00 & 0,75 & 9,22 & 1,64 & 0,00 & 0,72 & 0,00 & 3,11 & 0,00 & 0,42 \\
\hline Morcín & 0,00 & 0,00 & 8,97 & 0,00 & 0,00 & 0,00 & 0,00 & 0,00 & 0,00 & 0,00 & 8.97 & 0,00 \\
\hline Muros de & 0,00 & 5,79 & 0,00 & 0,00 & 0,00 & 0,00 & 0,00 & 0,00 & 0,00 & 5,79 & 0,00 & 0,00 \\
\hline Nava & 0,16 & 5.54 & 0,00 & 0,00 & 0,00 & 5,71 & 0,00 & 0,00 & 0,00 & 5.71 & 0.00 & 0,00 \\
\hline Navia & 0,75 & 0,67 & 2,21 & 1.06 & 0,00 & 0,00 & 5,28 & 1,14 & 0,00 & 1.42 & 0,00 & 1,24 \\
\hline Noreña & 0,00 & 2,34 & 0,00 & 0,44 & 0,00 & 3,07 & 0,00 & 0,00 & 0,00 & 1,53 & 0,00 & 0,92 \\
\hline Oviedo & 5,91 & 3,79 & 0,82 & 0,77 & 11.55 & 1.83 & 0.22 & 0.97 & 1.22 & 1.90 & 2,05 & 0,79 \\
\hline Parres & 1.01 & 3,21 & 0,00 & 0.79 & 1.53 & 2.42 & 0,00 & 0.83 & 0.00 & 0,00 & 0,00 & 1.60 \\
\hline Peñamellera Alta & 1,47 & 10,61 & 0,00 & 0,00 & 1,75 & 6,68 & 0,00 & 0,00 & 0,00 & 30,67 & 0,00 & 0,00 \\
\hline Pesoz & 1.50 & 0,00 & 0,00 & 0,00 & 1.50 & 0,00 & 0,00 & 0,00 & 0,00 & 0,00 & 0,00 & 0,00 \\
\hline Piloña & 0,00 & 0,00 & 0,13 & 2,28 & 0,00 & 0,00 & 0,24 & 2,24 & 0,00 & 0,00 & 0,00 & 2,31 \\
\hline Ponga & 0,00 & 0,00 & 0,00 & 0,00 & 0,00 & 0,00 & 0,00 & 0,00 & 0,00 & 0,00 & 0,00 & 0,00 \\
\hline Pravia & 3,57 & 0,89 & 0,00 & 0,81 & 7.38 & 0,00 & 0,00 & 0.57 & 0,00 & 1,73 & 0,00 & 1,03 \\
\hline Ribadesella & 0,00 & 8,89 & 0,00 & 0,37 & 0,00 & 11,62 & 0,00 & 0,00 & 0,00 & 6,33 & 0,00 & 0,72 \\
\hline Salas & 1,10 & 0,00 & 0,00 & 1,54 & 0,55 & 0,00 & 0,00 & 2,05 & 0,00 & 0,00 & 0,00 & 0,00 \\
\hline San Martín del Rey Aurelio & 0,00 & 5.91 & 0,00 & 0,20 & 0,00 & 6.88 & 0,00 & 0,00 & 0,00 & 5,18 & 0.00 & 0.34 \\
\hline Siero & 0,00 & 2,83 & 0,34 & 0,64 & 0,00 & 2,82 & 0,62 & 0,60 & 0,00 & 2,79 & 0,00 & 0,70 \\
\hline Sobrescobio & 1,57 & 0,00 & 0,00 & 0,97 & 2,62 & 0,00 & 0,00 & 0,00 & 0,00 & 0,00 & 0,00 & 0,00 \\
\hline & 1,71 & 0,00 & 0,00 & 0,00 & 0,00 & 0,00 & 0,00 & 0,00 & 1.71 & 0,00 & 0,00 & 0,00 \\
\hline Soto del Barco & 5,33 & 0,00 & 0,00 & 0,69 & 0,00 & 0,00 & 0,00 & 0,00 & 0,00 & 0,00 & 0,00 & 2,08 \\
\hline Tapia de Casariego & 0,00 & 3,41 & 0,00 & 1,64 & 0,00 & 4,95 & 0,00 & 1,47 & 0,00 & 0,00 & 0,00 & 2,02 \\
\hline Taramundi & 0,00 & 5,83 & 0,00 & 0,00 & 0,00 & 5.83 & 0,00 & 0,00 & 0,00 & 0,00 & 0,00 & 0,00 \\
\hline Teverga & 0,00 & 0,00 & 0,00 & 1,97 & 0,00 & 0,00 & 0,00 & 0,00 & 0,00 & 0,00 & 0,00 & 1,97 \\
\hline Tineo & 0,19 & 4,79 & 0,00 & 0,63 & 0,23 & 5,46 & 0,00 & 0,32 & 0,00 & 1,82 & 0,00 & 1,98 \\
\hline Vegadeo & 0,00 & 13,16 & 0,00 & 0.77 & 0,00 & 23,44 & 0,00 & 0,00 & 0.00 & 11,09 & 0,00 & 0.92 \\
\hline Villanueva & 1,82 & 0,00 & 0,00 & 0,00 & 1.82 & 0,00 & 0,00 & 0,00 & 0,00 & 0,00 & 0,00 & 0,00 \\
\hline Villavicios & 0,77 & 0,00 & 3,67 & 0,65 & 0,00 & 0,00 & 0,00 & 0,00 & 0,28 & 0,00 & 4,12 & 0,73 \\
\hline
\end{tabular}

* A,B: Agricultura, ganadería, caza y selvicultura; pesca; C,D: Industria; F: Construcción y G a O: Servicios.

Fuente: Elaboración propia. 
